\title{
Characteristic imaging findings of the respiratory system in penguins with suspected aspergillosis in an aquarium
}

\author{
Megumi ITOH ${ }^{1)}$, Takahito TOYOTOME ${ }^{1)}$, Naoya MATSUMOTO' ${ }^{2}$, \\ Minoru OKAMOTO ${ }^{3)}$, Ken-ichi WATANABE ${ }^{1)}$ and Kazutaka YAMADA4)* \\ ${ }^{1)}$ Obihiro University of Agriculture and Veterinary Medicine, Inada-cho, Obihiro, Hokkaido 080-8555, Japan \\ ${ }^{2)}$ Noboribetsu Marine Park Nixe, Noboribetsu, Hokkaido 059-0492, Japan \\ 3)Rakuno Gakuen University, 582 Bunkyodai-Midorimachi, Ebetsu, Hokkaido 069-8501, Japan \\ 4)Azabu University, Sagamihara, Kanagawa 252-5201, Japan
}

\section{J. Vet. Med. Sci.}

82(9): 1260-1266, 2020

doi: 10.1292/jvms.20-0129

Received: 8 March 2020

Accepted: 1 July 2020

Advanced Epub:

13 July 2020

\begin{abstract}
Twenty penguins, including the King penguin (Aptenodytes patagonicus), Gentoo penguin (Pygoscelis papua), and African penguin (Spheniscus demersus), housed at an aquarium in Hokkaido, Japan, underwent regular health screening via blood test, and five penguins with suspected aspergillosis were extracted. In cases 1 and 2, a thickened membrane and/or fluid level and/or calcification in the air sac were observed on both radiography and computed tomography (CT). These two penguins died after 19 and 43 days, respectively. At the time the radiographic changes were observed, the disease had likely progressed to a point at which it was too late for recovery. Aspergillus fumigatus infection was confirmed by nucleotide sequence analysis in case 1. In case 3 , infiltration in the pulmonary parenchyma was observed on $C T$, and the infiltration disappeared following oral administration of itraconazole as diagnostic therapy for 8 months. In case 4, defects in the pulmonary parenchyma were observed only on CT. These defects remained unchanged in size for 7 months despite the lack of any treatment, and were not considered clinically significant. However, the blood antigen level in case 5 was increased, both radiography and CT were unremarkable. The combination of a screening blood test and CT examination could be useful clues for an early diagnosis of aspergillosis as well as for initiating treatment.
\end{abstract}

KEY WORDS: aspergillosis, Aspergillus fumigatus, computed tomography, early diagnosis, penguin

Penguins kept in zoos and aquariums often affect respiratory disorders, including aspergillosis or Mycobacterium avium infection. An aquarium in Hokkaido, Japan, had been concerned about death due to aspergillosis [20]. Thus, we focused our research on the incidence of aspergillosis in the aquarium with the goal of eradication and, also investigated the early detection of suspected aspergillosis. Avian aspergillosis is a respiratory infection caused by Aspergillus fumigatus, Aspergillus flavus, Aspergillus niger, Aspergillus terreus, and Aspergillus nidulans, with 95\% of cases caused by A. fumigatus [17]. Although aspergillosis in wild penguins is rare, it is a significant cause of death in zoos and aquariums. Economic and genetic loss is substantial in these facilities. General clinical signs include chronic debilitation, vocal change, and exercise intolerance; however, these clinical findings are not specific for aspergillosis [10]. When clinical signs appear, the pathological condition has already progressed, and it can be more challenging to treat these cases successfully [1]. Therefore, it is important to detect initial changes as early as possible, preferably before the onset of clinical signs. Screening blood test findings for avian aspergillosis include increases in white blood cell count, serum total protein (TP), aspartate transaminase, and creatine kinase and decreases in serum albumin (Alb) and the albumin-to-globulin ratio (A/G) $[3,5]$. Similar to clinical signs, these results are not specific for aspergillosis [1]. Aspergillus antigens and antibodies are also used to diagnose aspergillosis [5]. Aspergillus spp. is a ubiquitous fungus in the environment, and in most cases, it seems that aspergillosis develops when immune-compromised animals inhale large quantities of spores. Resulting in many birds being exposed to this potential pathogen without developing clinical disease, it is difficult to diagnose aspergillosis based on serum antigen or antibody levels alone. [17]. Endoscopy, radiography, and computed tomography (CT) for birds have previously been reported as tools that can be used for the diagnosis of aspergillosis [6, $11,14]$. Although endoscopic examination allows direct visualization of nodules and plaques in the air sac [16], it is employed cautiously in penguins because it is invasive [1]. Radiographic examination is noninvasive; however, radiographic lesions are only appreciated with affecting at least one-third of the lung, and thus, radiography is unlikely to yield a diagnosis until disease severity

*Correspondence to: Yamada, K.: kyamada@azabu-u.ac.jp

(02020 The Japanese Society of Veterinary Science

This is an open-access article distributed under the terms of the Creative Commons Attribution Non-Commercial No Derivatives (by-nc-nd) License. (CC-BY-NC-ND 4.0: https://creativecommons.org/licenses/by-nc-nd/4.0/) 
has progressed [13]. Although CT examination requires general anesthesia, this procedure is otherwise noninvasive, and it can provide diagnostic information in penguins. In the present study, radiographic and CT examinations in penguins with suspected aspergillosis were performed to allow early diagnosis, and characteristic CT findings were obtained.

\section{MATERIALS AND METHODS}

\section{Cases}

As a regular quarterly health check, blood parameters were evaluated for 20 penguins (King penguin, Aptenodytes patagonicus, $\mathrm{n}=7$; Gentoo penguin, Pygoscelis papua, $\mathrm{n}=8$; and African penguin, Spheniscus demersus, $\mathrm{n}=5$ ) housed at an aquarium in Hokkaido, Japan. Five penguins (three King penguins and two Gentoo penguins) were suspected of aspergillosis after blood test (i.e., decrease in A/G, increase in Aspergillus antigen or antibody, Table 1). Later, two of these penguins died (cases 1 and 2) and were submitted for postmortem evaluation, and a polymerase chain reaction (PCR) test to identify the fungal species was performed. The health check and all diagnostic and treatment procedures were conducted in accordance with practice guidelines of the veterinary department of the aquarium.

Blood was sampled from the brachial vein or medial metatarsal vein, and the levels of TP, Alb, A/G, Aspergillus antigens, and Aspergillus antibody were measured. From previous reports and the results of the health check at the aquarium [2,8], penguins with an A/G ratio $\leq 0.8$, Aspergillus antigen (galactomannan index) $\geq 0.5$, or Aspergillus antibody titer determined by a complement fixation test (the highest dilution of serum) $\geq 1: 16$ were identified as suspected cases of aspergillosis and were then subjected to radiographic and CT examination.

\section{Radiography}

With the penguin in a standing position without sedation, lateral and ventrodorsal radiographs using digital radiography (Aero DR, Konica-Minoluta, Tokyo, Japan) were obtained. The imaging technique parameters were $70 \mathrm{kV}$ and $1.5 \mathrm{mAs}$. Digital Imaging and Communications in Medicine (DICOM) data were sent to a viewer (Newton OsiriX, Newton-Graphics, Sapporo, Japan) to allow evaluation of the radiographs.

\section{$C T$}

The nose and beak of the penguin were covered with a custom-made mask, and induced with $5 \%$ isoflurane and maintained $2 \%$ isoflurane for general anesthesia. After confirmation of the immobilization of the penguin, CT images were obtained with the penguin in the supine position using a four-row multidetector CT scanner (Asteion Super Four, Canon, Tokyo, Japan) with $80 \mathrm{kV}$, $150 \mathrm{~mA}, 0.75 \mathrm{sec} /$ rotation, and $1.0 \mathrm{~mm}$-slice thickness. DICOM data were sent to the viewer and read in the transverse, sagittal, and dorsal planes. The volume of all of the air sacs was measured using an image-processing workstation (Virtual Place, AZE, Kawasaki, Japan).

\section{Fungal species identification}

Fungal identification was performed as described previously [7, 15]. Briefly, DNA extraction from formalin-fixed paraffinembedded tissues of the agglomerate/nodule in the air sac with presumed fungal disease was performed using the NucleoSpin FFPE kit (Takara Bio Inc., Kusatsu, Japan). The prepared DNA was used for PCR amplification. The internal transcribed spacer (ITS) region and D1/D2 region of the large subunit of the 28S ribosomal RNA gene were amplified and sequenced with ITS4 (5'-TCCTCCGCTTATTGATATGC-3')/ITS5 (5'-GGAAGTAAAAGTCGTAACAAGG-3') primers [18] and NL1 (5'-GCATATCAATAAGCGGAGGAAAAG-3')/NL4 (5'-GGTCCGTGTTTCAAGACGG-3') primers [9], respectively. Because no amplicons were detected in the first PCR, a second PCR was performed under the same conditions. After PCR amplification, amplicons were purified and sequenced with each primer as detailed above.

\section{RESULTS}

\section{Case 1}

Radiographic and CT examination were performed due to an initial decrease in A/G (0.7) that progressed to an additional decrease in A/G (0.17) coupled with an increased Aspergillus antigen (1.0) on recheck. Coughing was observed at the time. Fluid

Table 1. Description of penguins extracted for aspergillosis diagnostic screening by blood test and performed radiographic and computed tomography

\begin{tabular}{lccccl}
\hline & Species & Sex & Age (years) & Body weight (kg) & Blood test \\
\hline Case 1 & King & Female & 2 & 10.4 & A/G $\downarrow$, Aspergillus antigen $\uparrow$ \\
Case 2 & King & Female & 23 & 11.2 & A/G $\downarrow$ \\
Case 3 & Gentoo & Female & 7 & 5.7 & Aspergillus antibody $\uparrow$ \\
Case 4 & Gentoo & Female & 8 & 5.3 & Aspergillus antibody $\uparrow$ \\
Case 5 & King & Male & 13 & 14.8 & Aspergillus antigen $\uparrow$ \\
\hline
\end{tabular}




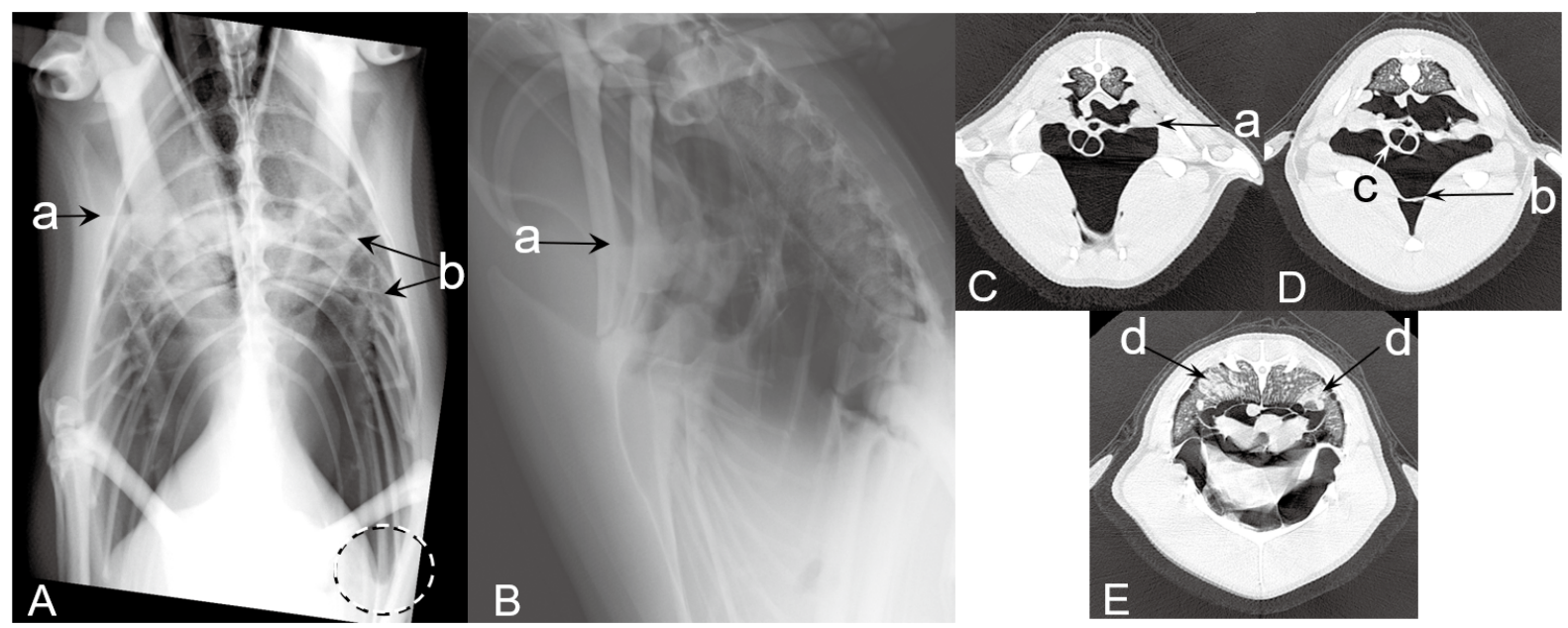

Fig. 1. Case 1 (King penguin): Radiographs were obtained in the standing position (A and B), computed tomography (CT) were obtained under anesthesia in the supine position (C, D, and E). The fluid level of the clavicular air sac (a), thickening membrane (b), and rounded edge of the air sac (the dashed circle) observed on radiographs and CT images. Thickening of the trachea wall (c) and infiltrate in the pulmonary parenchyma (d) observed only on the CT images.

in the clavicular air sac, thickening of the air sac membrane, and rounded edges of the air sac were observed on the radiographs (Fig. 1). On the CT images, fluid in the clavicular air sac and thickening of the air sac membrane were observed, which was consistent with the radiographic findings. In addition, thickening of the tracheal wall and infiltration in the pulmonary parenchyma were observed, which were not detected in the radiographs (Fig. 1). The volume of all air sacs as measured from the CT data was $828 \mathrm{~cm}^{3}$. The penguin died 19 days after imaging. The pathological findings included milky fluid accumulation, thickening of the bronchial wall, yellowish-white agglomerate in the air sac, and thickening of the air sac. Fungal infection was confirmed by Grocott staining of the paraffin-embedded tissue of the air sac (Fig. 2). The ITS region and the D1/D2 region of the fungal DNA obtained from the air sac tissues were amplified. The D1/D2 sequence was $100 \%$ identical to $A$. fumigatus, and the ITS sequence showed the highest bit score against $A$. fumigatus on a BLAST result. Therefore, case 1 was definitively diagnosed as aspergillosis.

\section{Case 2}

Radiographic and CT examinations were performed due to a decreased A/G (0.75) in the blood test. Both Aspergillus antigen and antibody were negative. In the radiographs, dilation of the right clavicular air sac and thickening of the membrane were present, and an area of opacification and nodules in the clavicular air sac were observed. On the CT images, thickening of the membrane and calcification were present, which were consistent with the radiographic findings (Fig. 3). Nodules in the air sac were observed in both radiographs and CT images (Fig. 4). The volume of all air sacs measured from the CT data was $974 \mathrm{~cm}^{3}$. Recheck blood test results at 6 months revealed an A/G of 0.79, Aspergillus antigen of 0.2, and Aspergillus antibody of $<1: 4$. The penguin died 43 days after CT examination. The pathological findings included yellowish nodules that were scattered in the air sac and the tracheal wall. Although Grocott staining of the tissue confirmed some fungi in the air sacs, nucleotide sequence analysis indicated that the amplified DNA fragments were non-pathogenic fungus and not genes derived from Aspergillus. This case was finally diagnosed with airsacculitis.

\section{Case 3}

Radiographic and CT examinations were performed because the Aspergillus antibody titer was increased to 1:32 in the blood test. Radiographs were unremarkable; however, infiltration in the pulmonary parenchyma was observed on CT imaging (Fig. 5A). A presumptive diagnosis of aspergillosis was made based on the high prevalence of aspergillosis in this aquarium, increased Aspergillus antibody in blood test, and CT findings. As a diagnostic therapy, oral administration of itraconazole was started. Seven days after treatment had started, the blood test results revealed that the Aspergillus antibody titer had decreased (1:4), but the Aspergillus antigen had increased (1.0). In the blood test repeated after 4 months of treatment, the Aspergillus antigen had decreased to 0.1 , and the antibody titer remained at 1:4. Upon CT at that time, the infiltration was reduced (Fig. 5B). After 8 months of treatment, blood test results showed that the Aspergillus antigen remained at 0.1 and the antibody titer had increased to $1: 8$, but the infiltration had completely disappeared (Fig. 5C). Oral administration of itraconazole was discontinued. On CT examination after 20 months (data not shown), no infiltration was observed, and no abnormality was observed in the blood test. This penguin remained alive.

\section{Case 4}

Radiographic and CT examinations were performed because the Aspergillus antibody titer was increased (1:16) in the blood test. 

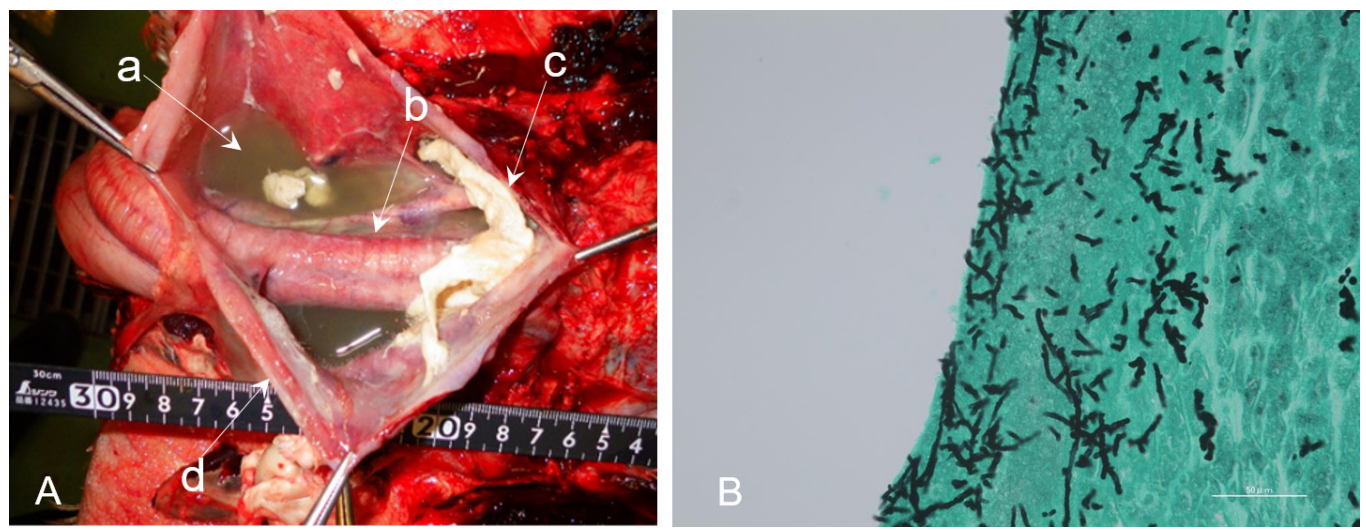

Fig. 2. Case 1 (King penguin): Gross pathologic examination (A) indicated milky fluid accumulation (a), thickening of the bronchial wall (b), yellowish-white agglomerate in the air sac (c), and thickening of the air sac (d). Fungal infection was confirmed by Grocott staining on histopathologic examination (B) of the agglomerate in the air sac.

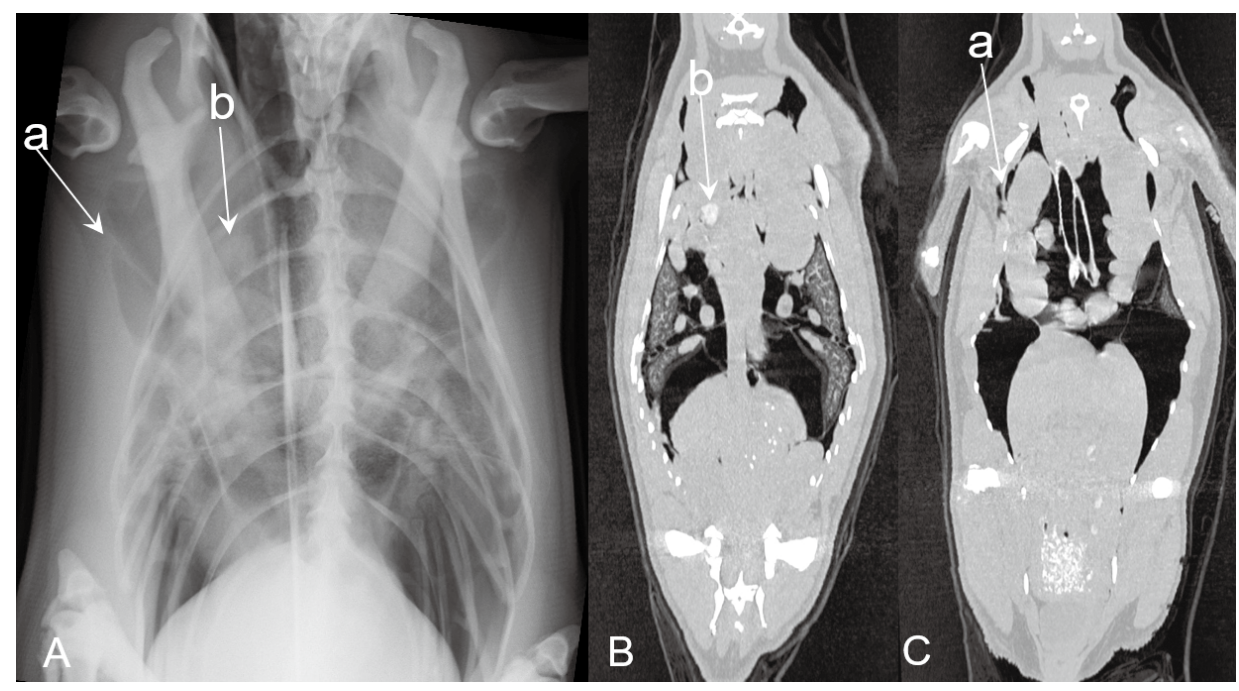

Fig. 3. Case 2 (King penguin): Radiographs were obtained in the standing position (A), computed tomography (CT) were obtained under anesthesia in the supine position (B and C). Dilation of the right clavicular air sac and thickening of the membrane (a) was observed in the radiograph, and calcification (b) was observed on radiographs and CT images.

Radiographs were unremarkable; however, defects of $11 \times 11 \mathrm{~mm}$ and $8 \times 8 \mathrm{~mm}$ in the right pulmonary parenchyma were observed on CT images (Fig. 6A). This case underwent follow-up observations without treatment. After 3 and 7 months, blood tests indicated that the antibody titer had decreased to 1:8 and 1:4, respectively. Upon CT examination after 7 months, no changes in the size of the defects were observed (Fig. 6B). This penguin remained alive.

\section{Case 5}

Radiographic and CT examinations were performed as a result of increased Aspergillus antigen (0.5) in the blood test. Radiographs and CT images were unremarkable. Upon CT examination after 2 years, no specific finding was observed (data not shown). This penguin remained alive.

\section{DISCUSSION}

Radiographic and CT examinations were performed on penguins with suspected aspergillosis identified through a blood screening test. In cases 1 and 2, a thickened membrane and/or increased fluid levels in the air sac were observed in both radiographs and CT images. In addition, the volume of the all air sacs on CT measurement was less than 1,000 $\mathrm{cm}^{3}$; in our experience, this volume should be about $1,500 \mathrm{~cm}^{3}$ on healthy King penguins (data not shown). These two penguins died after 19 and 43 days, respectively. When radiographic changes were observed, the disease had progressed to a point at which it was 


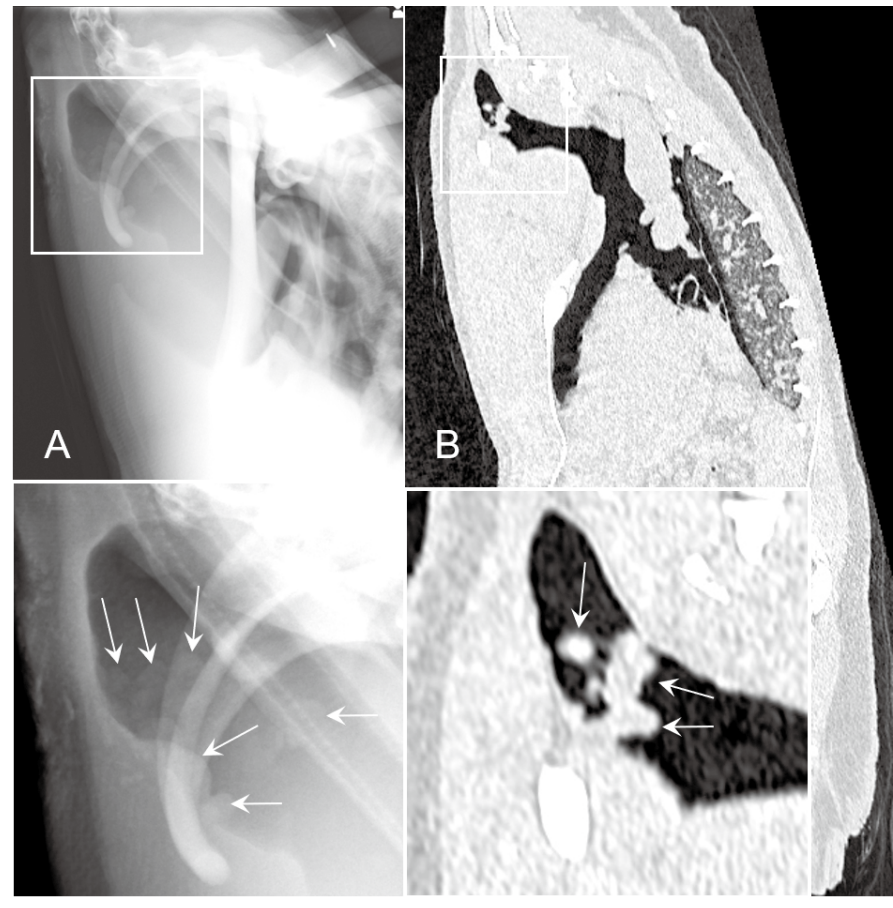

Fig. 4. Case 2 (King penguin): Radiographs were obtained in the standing position (A); computed tomography (CT) were obtained under anesthesia in the supine position (B). Nodules (arrows shown on the magnified images) in the clavicular air sac observed on radiographs and $\mathrm{CT}$ images.

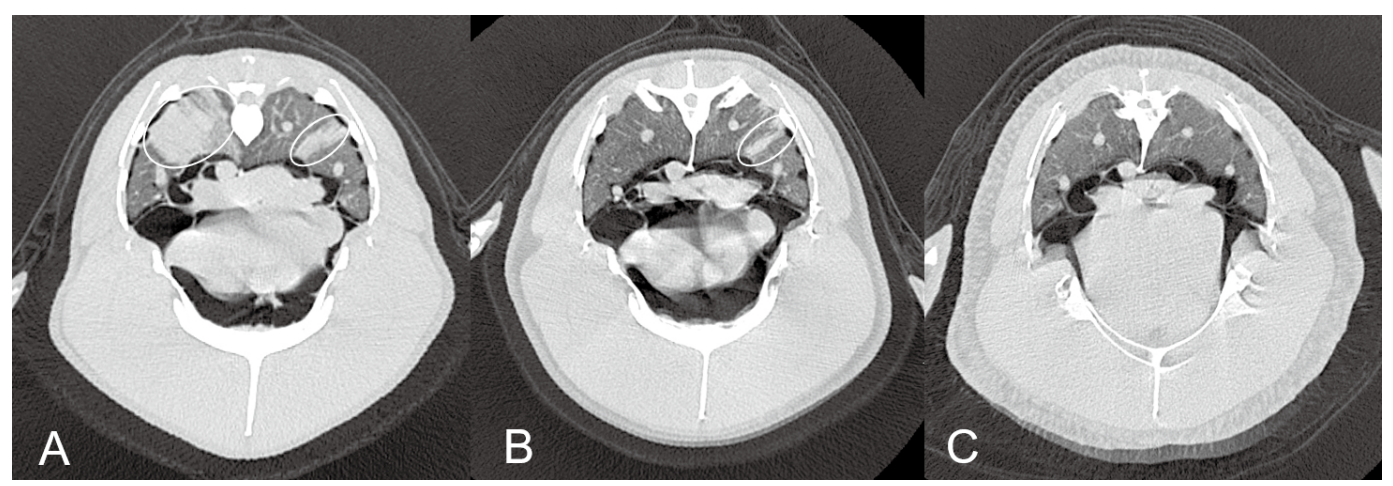

Fig. 5. Case 3 (Gentoo penguin): Computed tomography (CT) images (A, 0 months; B, 4 months; C, 8 months) showing infiltrations (circles) in the pulmonary parenchyma that gradually disappeared following administration of itraconazole over the time course.

too late to recover. The infiltration in the pulmonary parenchyma in case 1 and case 3 was detected only by CT, not radiography. Case 1 died, whereas case 3 survived. The observation of infiltration in CT would indicate incipient lesion, and if this infiltration is detected at an early stage and medical treatment is started, the affected penguins could be saved [3, 5]. Therefore, an early diagnosis in penguins is important to prevent death.

Case 1 was diagnosed as aspergillosis by nucleotide sequence analysis of the fungi, whereas case 2 was not. Although both cases had similar radiographic and CT findings, imaging cannot provide a definitive diagnosis of aspergillosis. As case 3 remains alive, it is difficult to confirm definitive diagnosis of aspergillosis because sampling for analysis is invasive. However, we strongly suspected that case 3 was affected by Aspergillus, because of the high prevalence of aspergillosis in this aquarium, both Aspergillus antibody and antigen blood levels were increased, and the specific findings of infiltration in the pulmonary parenchyma on CT images. Furthermore, the penguin responded to diagnostic therapy. As the antibody titer decreased, infiltration in the pulmonary parenchyma reduced. We believe that the penguin survived because itraconazole was administered prior to the onset of clinical signs.

Although CT examination of the penguins in this report was done under general anesthesia, the time to complete examination was less than $15 \mathrm{~min}$, and the procedure was not invasive. Therefore, CT is considered a less risky tool for follow-up examination. A report of CT without anesthesia in penguins was recently published [12], and CT examination without anesthesia provides an easy and safe diagnostic for assessing respiratory system disease in penguins.

There are findings of cavitary lesions in human aspergillosis. A cavitary lesion is surrounded by a thickened wall, and the size of the cavity increases as the condition progresses [4]. In case 4, no wall was observed around the defects, and despite the lack of 


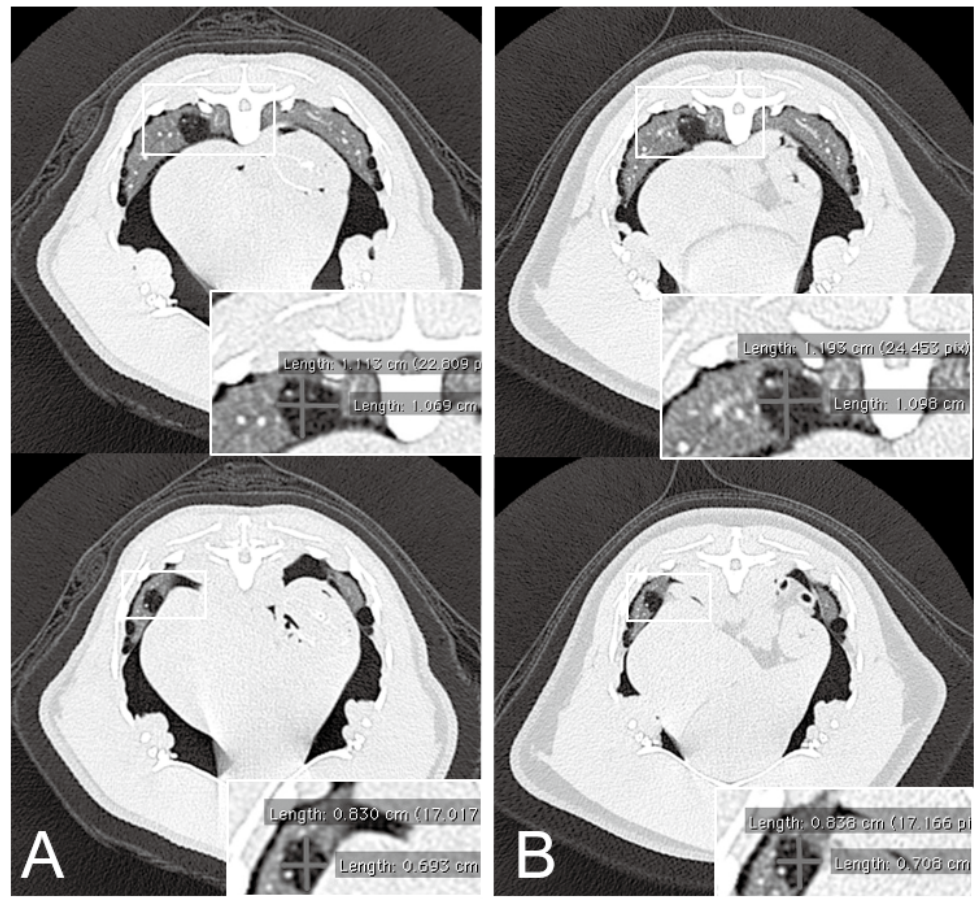

Fig. 6. Case 4 (Gentoo penguin): Computed tomography (CT) images (A, 0 months; B, 7 months) showing no changes in the size of the defects over a 7-month period. Because case 4 did not receive any treatment, the defects in the pulmonary parenchyma were not considered to be clinically significant.

treatment, there were no changes in the size of the defects after 7 months, and no clinical signs manifested. It has been reported that mycobacterial pulmonary nodules in the lungs of penguins change to an open defect following successful treatment with anti-mycobacterial medications [13]. The pulmonary defects of case 4 were considered not clinically significant based on the lack of outward evidence of difficulty breathing in spite of their presence.

The antibody titer of 1:32 in case 3 led to the suspicion of aspergillosis; however, the antibody titer of 1:16 in case 4 with defects in the pulmonary parenchyma was not considered clinically significant. The antigen level in case 5 increased in the blood test, but no specific findings were observed on either radiography or CT, and no clinical signs were present. It was difficult to determine aspergillosis in penguins by antigen or antibody testing alone. However, an antibody titer $>1: 32$ may be a factor in deciding whether to test CT examination.

In this aquarium, King penguins, Gentoo penguins, and African penguins were housed, and all species underwent screening blood tests. King and Gentoo penguins, but not African penguins, were found to have abnormal values in blood screening tests Of note, all penguins were born in Japan or had been kept for more than 20 years in this aquarium, but the habitat of the King penguins and Gentoo penguins is the polar region (i.e., Antarctic Sea or Sub-Antarctic), whereas the habitat of the African penguins is a temperate zone (i.e., coast of South Africa). Aspergillosis has occurred in the habitat of African penguins [19]. It is the authors thought that among wild African penguins, which have been exposed to Aspergillus during the process of evolution, those that are not tolerant to aspergillosis have been eliminated by natural selection, and only the resistant penguins have survived. For wild King and Gentoo penguins, which may be less exposed to Aspergillus in their natural environment, there has been less opportunity to acquire resistance to Aspergillus during the process of evolution, and they therefore develop aspergillosis more easily in captivity. In addition, although Hokkaido is a relatively cool region of Japan, it is substantially hotter than the penguins' natural habitat. Heat stress on the King and Gentoo penguins is also considered to be a factor that may contribute to the development of aspergillosis.

In any zoo or aquarium, countermeasures for aspergillosis in penguins are important in order to prevent economic and genetic loss. In some aquariums in Japan, the enclosures are cleaned to prevent aspergillosis. Cleaning of the environment is important, but because aspergillosis is an opportunistic infection that acts through immune depression, it is difficult to prevent it completely. In other aquariums in Japan, antifungal agents are administered prophylactically as a countermeasure for aspergillosis; however, the administration of these agents to all penguins involves high cost and carries the risk of the development of drug resistance.

The combination of a screening blood test and CT examination could be useful clues for an early diagnosis of aspergillosis as well as for initiating treatment. The characteristic CT findings described herein could help provide an early diagnosis of aspergillosis in suspected penguins.

ACKNOWLEDGMENT. The authors would like to express sincere gratitude to the aquarists of Noboribetsu Marine Park NIXE for their assistance in sampling. This work was supported by the JSPS Grant-in-Aid for Scientific Research (C) Number 17 K08095. 


\section{REFERENCES}

1. Beernaert, L. A., Pasmans, F., Van Waeyenberghe, L., Haesebrouck, F. and Martel, A. 2010. Aspergillus infections in birds: a review. Avian Pathol. 39: 325-331. [Medline] [CrossRef]

2. Cray, C., Watson, T., Rodriguez, M. and Arheart, K. L. 2009. Application of galactomannan analysis and protein electrophoresis in the diagnosis of aspergillosis in avian species. J. Zoo Wildl. Med. 40: 64-70. [Medline] [CrossRef]

3. Fischer, D. and Lierz, M. 2015. Diagnostic procedures and available techniques for the diagnosis of aspergillosis in birds. J. Exot. Pet Med. 24: 283-295. [CrossRef]

4. Godet, C., Laurent, F., Bergeron, A., Ingrand, P., Beigelman-Aubry, C., Camara, B., Cottin, V., Germaud, P., Philippe, B., Pison, C., Toper, C., Carette, M. F., Frat, J. P., Béraud, G., Roblot, F., Cadranel J., ACHROSCAN Study Group. 2016. CT imaging assessment of response to treatment in chronic pulmonary aspergillosis. Chest 150: 139-147. [Medline] [CrossRef]

5. Jones, M. P. and Orosz, S. E. 2000. The Diagnosis of Aspergillosis in birds. Semin. Avian Exot. Pet Med 9: 52-58. [CrossRef]

6. Krautwald-Junghanns, M., Schumacher, F. and Tellhelm, B. 1993. Evaluation of the lower respiratory tract in pisttacines using radiology and computed tomography. Vet. Radiol. Ultrasound 34: 382-390. [CrossRef]

7. Nakagun, S., Okazaki, M., Toyotome, T., Sugiyama, N., Watanabe, K., Horiuchi, N. and Kobayashi, Y. 2018. Fatal pulmonary and cerebellar zygomycosis due to Rhizomucor pusillus in a ringed seal (Pusa hispida). Mycopathologia 183: 979-985. [Medline] [CrossRef]

8. Naylor, A. D., Girling, S. J., Brown, D., Crompton, C. G. and Pizzi, R. 2017. Plasma protein electrophoresis as a prognostic indicator in Aspergillus species-infected Gentoo penguins (Pygoscelis papua papua). Vet. Clin. Pathol. 46: 605-614. [Medline] [CrossRef]

9. O’Donnell, K. 1993. Fusarium and its near relatives. pp. 225-233. In: The Fungal Holomorph: Mitotic, Meiotic and Pleomorphic Speciation in Fungal Systematics. 1st ed. (Reynolds, D. R. and Taylor, J. W. eds.), CAB International, Wallingford.

10. Oglesbee, B. L. 1997. Mycotic diseases. pp. 323-361. In: Avian Medicine and Surgery. 1st ed. (Altman, R. B., Clubb, S. L., Dorrestein, G. M. and Quesenberry, K. eds.), W. B. Saunders Co, Philadelphia.

11. Phalen, D. N. 2000. Respiratory medicine of cage and aviary birds. Vet. Clin. North Am. Exot. Anim. Pract. 3: 423-452, vi. [Medline] [CrossRef]

12. Rivas, A. E., Fischetti, A. J., Le Roux, A. B., Hollinger, C., Oehler, D. A. and Paré, J. A. 2019. Standing computed tomography in nonanesthetized little penguins (Eudyptula minor) to assess respiratory system anatomy and monitor disease. J. Zoo Wildl. Med. 50: 396-404. [Medline] [CrossRef]

13. Rivas, A. E., Hollinger, C., Oehler, D. A., Robbe-Austerman, S. and Paré, J. A. 2019. Diagnosis and management of mycobacteriosis in a colony of little penguins (Eudyptula minor). J. Zoo Wildl. Med. 50: 427-436. [Medline] [CrossRef]

14. Schwarz, T., Kelley, C., Pinkerton, M. E. and Hartup, B. K. 2016. Computed tomographic anatomy and characteristics of respiratory aspergillosis in juvenile whooping cranes. Vet. Radiol. Ultrasound 57: 16-23. [Medline] [CrossRef]

15. Tanaka, Y., Toyotome, T., Inokuma, H., Watanabe, K. I., Kobayashi, Y. and Horiuchi, N. 2019. Rhinocerebral zygomycosis due to a Lichtheimia ramosa infection in a calf: neural spread through the olfactory nerves. Mycopathologia 184: 141-146. [Medline] [CrossRef]

16. Taylor, M. 1997. Endoscopic diagnosis of avian respiratory tract diseases. Semin. Avian Exot. Pet Med 6: 187-194. [CrossRef]

17. Tell, L. A. 2005. Aspergillosis in mammals and birds: impact on veterinary medicine. Med. Mycol. 43 Suppl 1: S71-S73. [Medline] [CrossRef]

18. White, T. J., Bruns, T., Lee, S. and Taylor, J. 1990. Amplification and direct sequencing of fungal ribosomal RNA genes for phyloge- netics. pp. 315-322. In: PCR Protocols: A Guide to Methods and Applications (Innis, M. A., Gelfand, D. H., Sninsky, J. J. and White, T. J. eds.), Academic Press, San Diego.

19. Yabsley, M. J., Parsons, N. J., Horne, E. C., Shock, B. C. and Purdee, M. 2012. Novel relapsing fever Borrelia detected in African penguins (Spheniscus demersus) admitted to two rehabilitation centers in South Africa. Parasitol. Res. 110: 1125-1130. [Medline] [CrossRef]

20. Yamada, K., Toyotome, T., Matsumoto, N. and Itoh, M. 2020. Autopsy imaging for aspergillosis in King Penguin, an economically valuable zoo animal. J. Vet. Med. Sci. 82: 373-375. [Medline] [CrossRef] 